\title{
Controlling the group velocity of an intense laser pulse using a pre-pulse
}

\author{
Samuel R. Yoffe ${ }^{\mathrm{a}}$, Bernhard Ersfeld ${ }^{\mathrm{a}}$, Devki N. Gupta ${ }^{\mathrm{b}}$, Arohi Jain ${ }^{\mathrm{b}}$, Matthew P. Tooley ${ }^{\mathrm{a}, \mathrm{c}}$, \\ George K. Holt ${ }^{\mathrm{a}}$, and Dino A. Jaroszynski ${ }^{\mathrm{a}}$ \\ aDepartment of Physics, SUPA \& University of Strathclyde, Glasgow G4 0NG (UK) \\ ${ }^{b}$ Department of Physics and Astrophysics, University of Delhi, Delhi 110007 (India) \\ ${ }^{\mathrm{c} N u m e r i c a l ~ A l g o r i t h m s ~ G r o u p ~ L t d ., ~ M a n c h e s t e r ~(U K) ~}$
}

\begin{abstract}
The accelerating structure of the laser wakefield accelerator (LWFA) is dynamic and highly sensitive to the local laser and plasma properties. It can expand and contract as it responds to the evolution of the laser and plasma fields. As a result, the position of, and environment within, the LWFA bubble are usually time dependent, which is not ideal for stable acceleration. Variations can have a negative impact on electron bunch properties, and are deleterious for ion channel lasers and plasma wigglers. We demonstrate how a laser pre-pulse improves the stability of the LWFA, and controls the evolution of the laser group and bubble velocity, which are important for determining LWFA dephasing and ultimately the electron bunch energy.
\end{abstract}

Keywords: Laser wakefield accelerator, LWFA, electron acceleration, laser group velocity, numerical simulation, particle-in-cell, PIC

\section{INTRODUCTION}

The laser wakefield accelerator (LWFA) was proposed in the late 1970s by Tajima and Dawson. ${ }^{1}$ The ponderomotive force of a short, intense laser pulse is used to displace electrons in plasma to create extremely high space-charge fields arising from charge separation. The restoring force attracts electrons back towards the laser axis, around which they oscillate at their local plasma frequency while accelerating.

At high laser intensities, the displaced electrons define a thin, high density sheath that surrounds a region of very low electron density. The transverse and longitudinal fields within this ion-dominated bubble drive electrons towards its centre and can be orders of magnitude greater than accelerating fields in conventional accelerator radiofrequency (RF) cavities. Electrons captured (by a process known as injection) at the back of the bubble are rapidly accelerated to very high energies. This can be achieved by injecting an electron beam from an external source, by ionising atoms within the bubble (ionisation injection), or by self-injection of electrons from the bulk plasma (which can be stimulated ${ }^{2,3}$ ). In the latter case, electrons crossing at the back of an evolving bubble find themselves inside the bubble, where they experience very high electric fields and rapidly accelerate to high energies. This can be considered as a form of wavebreaking.

Controlled acceleration of self-injected electrons was first demonstrated in $2004 .^{4-6}$ Since then, LWFAs have been used to produce high energy ${ }^{7-9}$ electron bunches with excellent properties. ${ }^{10-13}$ They have found wide ranging uses as compact X-ray ${ }^{14}$ and $\gamma$-ray ${ }^{15}$ sources with many potential applications. ${ }^{16-18}$ LWFAs are being developed for radiotherapy and medical imaging ${ }^{19-22}$ and as ultra-compact synchrotrons, free-electron lasers ${ }^{23-25}$ and ion channel lasers (ICLs). ${ }^{26,27}$

A Langmuir wave (and its associated wakefield), driven by an intense laser pulse, is often considered as trailing it at the group velocity of the laser pulse in undisturbed plasma, which is less than the speed of light in vacuum:

$$
\beta_{g}\left(n_{0}, \lambda_{0}\right)=\frac{v_{g}}{c}=\sqrt{1-\frac{n_{0}}{n_{\text {crit }}\left(\lambda_{0}\right)}}<1 \quad \text { with } \quad n_{\text {crit }}\left(\lambda_{0}\right)=m_{e} \epsilon_{0}\left(\frac{2 \pi c}{e \lambda_{0}}\right)^{2},
$$

Further author information: (Send correspondence to S.R.Y. or D.A.J.)

S.R.Y.: E-mail: sam.yoffe@strath.ac.uk

D.A.J.: E-mail: d.a.jaroszynski@strath.ac.uk 
where $c$ is the speed of light in vacuum, $n_{0}$ is the plasmas density, $n_{\text {crit }}\left(\lambda_{0}\right)$ is the critical density for laser wavelength $\lambda_{0}, m_{e}$ is the mass of the electron, $\epsilon_{0}$ is the vacuum permittivity, and $e$ is the fundamental charge. As $\beta_{g}<1$, the rapid acceleration of electrons in the LWFA quickly takes their velocity above this, $\beta_{e} \approx 1>\beta_{g}$. They therefore move forward in the accelerating structure, experiencing progressively weaker accelerating fields until they reach the dephasing point close to the centre of the bubble. Beyond this point, electrons enter the decelerating phase of the LWFA, where they lose energy. The resulting energy chirp can be used to ballistically compress the electron bunch by allowing lower energy electrons at the rear to catch up with electrons at the front, which reduces the bunch size.

As the laser propagates in plasma, the front of the pulse loses its energy to the wakefield, know as laser etching. This causes the bubble to travel at a reduced velocity,

$$
\beta_{\mathrm{g}}^{\prime}\left(n_{0}, \lambda_{0}\right)=\beta_{\mathrm{g}}\left(n_{0}, \lambda_{0}\right)-\frac{1}{\gamma_{g}^{2}\left(n_{0}, \lambda_{0}\right)},
$$

where $\gamma_{g}^{2}\left(n_{0}, \lambda_{0}\right)=n_{\text {crit }}\left(\lambda_{0}\right) / n_{0}$ is the Lorentz factor associated with the laser group velocity. The reduced group velocity is used to estimate the dephasing length and the maximum energy achievable in a LWFA. This is calculated by considering the time it takes for electrons entering at the back of the bubble to reach the dephasing point, given that the relative velocity is $\Delta \beta \approx 1-\beta_{\mathrm{g}}^{\prime}$. If the length from the back of the bubble to the dephasing point is defined as $L_{d}$ and the longitudinal electric field has a magnitude $E_{z}$, then naïvely the maximum electron energy is

$$
E_{\max }=\frac{e L_{d} E_{z}}{\Delta \beta}
$$

It is common to take the approximate bubble radius, $L_{d}=R\left(n_{0}, a_{0}\right)$, as the length when calculating dephasing and the energy limit, where

$$
R\left(n_{0}, a_{0}\right)=\lambda_{p}\left(n_{0}\right) \sqrt{a_{0}} / \pi
$$

with the plasma wavelength for density $n_{0}$ given by $\lambda_{p}\left(n_{0}\right)$ and the normalised peak laser amplitude $a_{0} \approx 8.55 \times 10^{-6} \lambda \sqrt{I_{\mathrm{rms}}}$. In practice, many factors prevent this limit from being respected, including laser depletion, beam loading, and non-linear evolution of the laser and bubble parameters.

In this article, we investigate the evolution of the laser group velocity and its implications for the LWFA maximum energy and electron bunch quality. In Section 2 we discuss the evolution of the laser group velocity determined from numerical simulations of the LWFA. We then introduce a new scheme for enhancing the stability of the accelerating structure in Section 3 and estimate the dephasing length and maximum energy possible from the accelerator. Conclusions are presented in Section 4.

\section{EVOLUTION OF THE LASER GROUP VELOCITY}

When discussing the propagation of a laser pulse in plasma, it is common to use equation (1) with initial parameters to estimate the group velocity. However, this neglects three important points:

- the critical density is only determined for a particular wavelength;

- electrons oscillating in an intense laser field gain a relativistic mass shift; and

- the front of the laser disturbs the local plasma density encountered by the rest of the pulse.

We redefine the critical density and group velocity to address these shortcomings,

$$
\beta_{g}\left(n_{e}, \lambda, a_{0}\right)=\sqrt{1-\frac{n_{e}}{n_{\text {crit }}\left(\lambda, a_{0}\right)}} \quad \text { with } \quad n_{\text {crit }}\left(\lambda, a_{0}\right)=\gamma\left(a_{0}\right) m_{e} \epsilon_{0}\left(\frac{2 \pi c}{e \lambda}\right)^{2},
$$

where $n_{e}$ is the instantaneous electron density experienced by the laser, $\gamma \approx \sqrt{1+a_{0}^{2} / 2}$ is the Lorentz factor associated with the relativistic mass shift, and $\lambda$ is the central wavelength. We explore the impact of these considerations using results from particle-in-cell simulations performed using FBPIC, ${ }^{28}$ an open-source, GPU-based, 

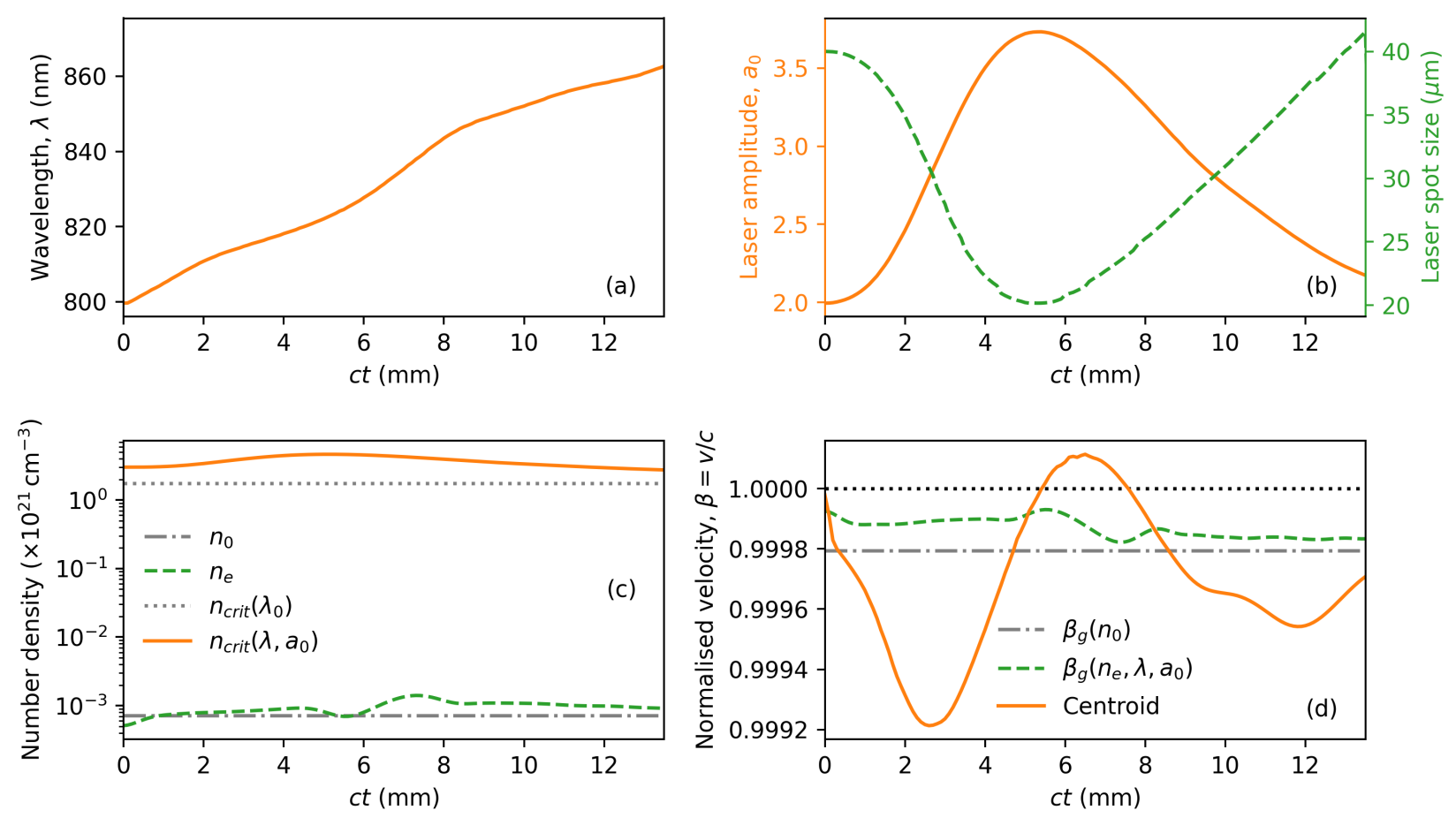

Figure 1. Evolution of the LWFA. Part (a) shows the increase of the laser wavelength as it propagates through the plasma. Part (b) shows the evolution of the laser amplitude (orange, solid line) and spot size $\left(1 / e^{2}\right.$ intensity; green, dashed line), indicating self-focussing. Part (c) compares the actual density at the laser centroid (green, dashed line) with the unperturbed background density, $n_{0}$ (grey, dot-dash line). It also compares the instantaneous critical density (orange, solid line) calculated from equation (5) during the propagation with the critical density given by equation (1) for the initial parameters (dotted line). Part (d) compares the velocity of the laser centroid (orange, solid line) with the instantaneous group velocity (green, dashed line) from (5) and the value given by equation (1) (grey, dot-dash line).

quasi-3D code. Particles are free to move in 3D, but cylindrical geometry and azimuthal modal decomposition are used to evaluate the fields, which significantly reduces the resources required. Simulations are performed for a linearly-polarised $800 \mathrm{~nm}$ laser, with initial amplitude $a_{0}=2\left(I_{\mathrm{rms}}=8.55 \times 10^{18} \mathrm{~W} / \mathrm{cm}^{2}\right)$, full-width halfmaximum (FWHM) intensity duration $45 \mathrm{fs}$ and focal spot size $40 \mu \mathrm{m}$. This propagates in uniform plasma with density $n_{0}=7.17 \times 10^{17} \mathrm{~cm}^{-3}$. The simulation uses grid cells of $20 \mathrm{~nm} \times 100 \mathrm{~nm}$ with $(3,2,12)$ particles per cell in $(z, r, \theta)$.

Figure 1(a) shows the evolution of the central wavelength as the laser propagates in the plasma. We observe that the wavelength increases as the laser photons decelerate, ${ }^{29}$ which reduces both the critical density and group velocity.

The relativistic mass shift observed is due to momentum gained from the laser fields, which depends on the laser amplitude, $a_{0}$. Figure 1(b) shows the evolution of the laser amplitude (in orange). It is observed that the laser undergoes relativistic self-focussing as it propagates through the plasma - its amplitude increases and its spot size (dashed green) decreases. While this variation is slower than observed in higher density plasma,,$^{3,30}$ the amplitude nearly doubles, which has a significant influence on the critical density and therefore the group velocity.

As the laser propagates in the plasma it displaces electrons. This leads to an increase in the distal density (snowplough effect), while a reduction of the anterior density, to the extent that the laser pulse does not really experience the background plasma density, $n_{0}$. This can lead to pulse compression, since the rear of the pulse experiences lower density and travels faster than the front. However, this is compensated for by the transverse 
density profile, which can act as a waveguide. The disperation relation for a laser in plasma can be written

$$
\omega^{2}=k^{2} c^{2}+\omega_{p}^{2},
$$

and for a laser in a (vacuum) waveguide as

$$
\omega^{2}=k^{2} c^{2}+\left(\frac{c}{\ell}\right)^{2},
$$

with $\omega$ and $k$ the laser frequency and wavenumber, $\omega_{p}$ the plasma frequency, and $\ell$ the lengthscale of the waveguide. When the waveguide scale is similar to the plasma wavelength, $\ell \sim \lambda_{p}$, as is the case for the bubble, then $(c / \ell)^{2} \sim \omega_{p}^{2}$ and the rear of the laser in the low density plasma (even vacuum) propagates at close to the group velocity in the unperturbed plasma, reducing pulse compression.

Figure 1(c) shows (dashed green line) the evolution of the on-axis density at the laser centroid position - the weighted centre of the intensity distribution. This should be compared with the line indicating $n_{0}$ (dot-dashed grey), from which we observe that the laser centroid usually sits in a region of increased electron density. The figure also shows the evolution of the calculated critical density (in orange) including the temporal variation of the laser wavelength and amplitude. This is noticeably higher than the critical density calculated using equation (1) (dotted line).

Finally, in figure 1(d) we compare the predicted evolution of the group velocity using equations (1) and (5) with the velocity of the laser centroid extracted from the simulation data (orange). This is often used as a surrogate for the effective group velocity of the laser. We see that the corrected expression for the group velocity (dashed green) shows a significant increase in the group velocity, compared with equation (1) indicated by the

dot-dash line. Most striking is the large variation of the centroid velocity, which initially drops well below the predicted value, and significantly reduces the time that injected electrons take to reach dephasing. But it then increases and is even briefly superluminal.

The evolution of the laser centroid velocity observed in figure 1(d), and its amplitude and spot size in part (b), highlight a general issue with LWFAs: a significant (and often rapid) evolution of the laser properties ${ }^{31}$ has serious implications for the stability of the accelerating structure, and the maximum electron energy and bunch quality achievable. ${ }^{32}$ It also has an impact on the suitability of using the bubble as an ion cavity for ion channel lasers and plasma wigglers. The length and radius of the bubble (and consequently the accelerating and focussing fields), and its relative position behind the laser pulse, depend strongly on the laser amplitude and propagation velocity. These properties vary and result in a highly dynamic accelerating structure that is not ideal for producing high energy and high quality electron bunches.

\section{STABILITY ENHANCEMENT USING A LASER PRE-PULSE}

We now demonstrate how introducing an intense laser pre-pulse enhances the stability of laser and LWFA bubble evolution. An identical laser pulse to the main pulse described above, but with initial amplitude $a_{0}=1.35$ is fired ahead of the main pulse. Figure 2 illustrates the setup: the pre-pulse (green) arrives ahead of the main pulse (red) and drives a relatively low amplitude plasma wave (dashed grey). The main pulse is timed to arrive just in front of the first plasma wave crest. In these simulations the lasers have linear polarisations perpendicular to each other. This unique scheme is distinct from the long delay pre-pulses used to produce plasma waveguides ${ }^{33-40}$ or the resonant excitation of a plasma wave by a train of laser pulses. ${ }^{41-43}$

The pre-pulse is observed to have a stabilising effect on the main laser pulse, and consequently on the LWFA bubble that it produces. Figure 3(a) presents the evolution of the laser centroid velocity with (blue) and without (dashed orange) the pre-pulse. There is a significant reduction in the variation of the group velocity in the presence of the pre-pulse.

As well as the group velocity, the presence of the pre-pulse enhances the stability of the dephasing point and dephasing length. Figure 3(b) shows the evolution of the accelerating length, $L_{d}$, measured from the back of the bubble to the dephasing point with (blue) and without (dashed orange). The single pulse case (without the pre-pulse) exhibits alternating expansion and contraction of the bubble and length of the accelerating region, 


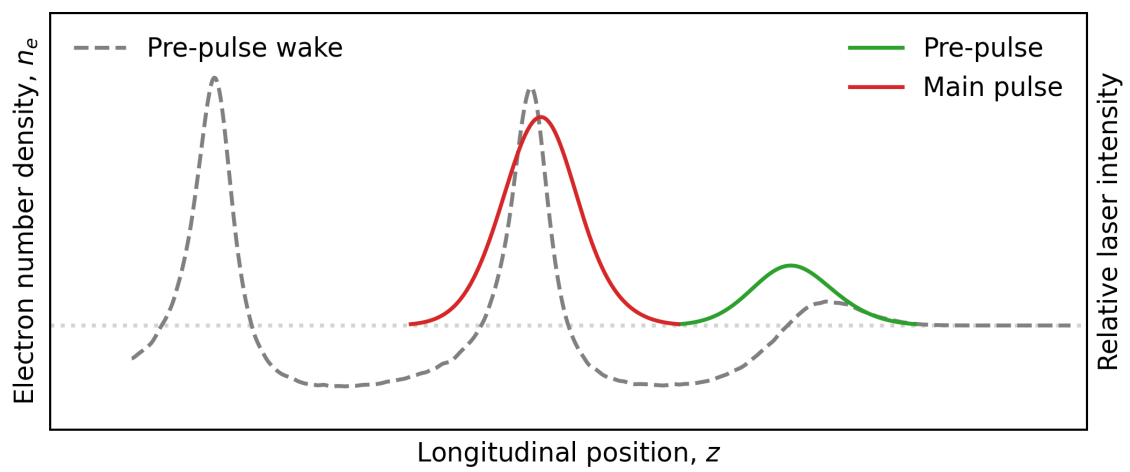

Figure 2. Illustration of the setup for the double pulse LWFA scheme. An intense pre-pulse (green, solid line) drives a low amplitude wakefield (grey, dashed line) that interacts with the main laser (red, solid line) that follows a short delay behind the pre-pulse.

whereas with the pre-pulse the accelerating length just slowly grows for $c t>4 \mathrm{~mm}$. This indicates that the bubble is more stable.

Figure 3(c) shows the evolution of the velocity of the dephasing point, $\beta_{d}$, extracted from the simulation data without the pre-pulse (dashed orange). We observe that $\beta_{d}$ varies significantly and, like the centroid velocity even becomes superluminal. This could be seen as advantageous for dephasing, but it also corresponds to the region where the bubble contracts [see part (b)] and the back of the bubble can move through the electron bunch, which can be disasterous for bunch quality as well as preventing further acceleration. We note that the value of $\beta_{d}$ is quite different from the reduced group velocity based on the initial parameters, $\beta_{\mathrm{g}}^{\prime}\left(n_{0}, \lambda_{0}\right)$, as given by equation (2) and indicated by the grey dot-dash line.

From the instantaneous values of the density, wavelength and amplitude, we can also define the instantaneous reduced group velocity

$$
\beta_{\mathrm{g}}^{\prime}\left(n_{e}, \lambda, a_{0}\right)=\beta_{\mathrm{g}}\left(n_{e}, \lambda, a_{0}\right)-\frac{1}{\gamma_{g}^{2}\left(n_{e}, \lambda, a_{0}\right)},
$$

which is also shown for comparison in Fig. 3(c). As seen for the group velocity in Fig. 2(d), the velocity of the dephasing point based on the instantaneous values is faster than the estimate based on the initial conditions.

Figure 3(d) shows the evolution of the dephasing length, $L_{d} / \Delta \beta$. Provided the laser and bubble remain well behaved, electrons injected at position $c t$ can be accelerated for the corresponding length. However, we have observed this not to be the case for the single pulse LWFA, as the bubble expands and contracts in response to the laser. We observe straight away that the projected dephasing length with the pre-pulse is much more stable, and actually in good agreement with that predicted using $\beta_{\mathrm{g}}^{\prime}\left(n_{0}, \lambda_{0}\right)$ and the length plotted in part (b). The increased instantaneous reduced group velocity given by equation (8) predicts a significantly longer dephasing length and therefore a much higher maximum energy for the electrons than could actually be obtained.

The dephasing lengths predicted are significantly longer than the length over which the simulations were performed, but we can estimate the maximum electron energy achievable with and without the pre-pulse. Without the pre-pulse, assuming the longitudinal field is linear within the bubble and that it is not degraded by the laser losing too much energy, the average field experienced by the electrons is $-59 \mathrm{GV} / \mathrm{m}$. An electron injected at $4 \mathrm{~mm}$ could be accelerated for $1.9 \mathrm{~cm}$ to reach an energy of $1.1 \mathrm{GeV}$. With the pre-pulse, the average field is $-62 \mathrm{GV} / \mathrm{m}$ and an electron injected at $4 \mathrm{~mm}$ can be accelerated for $3.35 \mathrm{~cm}$ to reach $2.1 \mathrm{GeV}$. These should be compared with the estimate of $\approx 1.75 \mathrm{GeV}$ obtained using the initial parameters, $L_{d}=R(2)$ and $\beta_{g}^{\prime}\left(n_{0}, \lambda 0\right)$.

In practice, the dynamic nature of the bubble will disrupt this limit. Figure 3(e) shows the separation between an electron at position $z(t)$ that was injected at $c t=4 \mathrm{~mm}$, and the position of the dephasing point, $z_{d}(t)$, calculated from the simulation data, where $\Delta z_{d}=z-z_{d}$. With the pre-pulse, we see that the electron slowly approaches dephasing, but that the slow expansion of the bubble [part (b)] extends the period of acceleration before dephasing is reached. Because the stability of the accelerating structure is enhanced by the pre-pulse, 

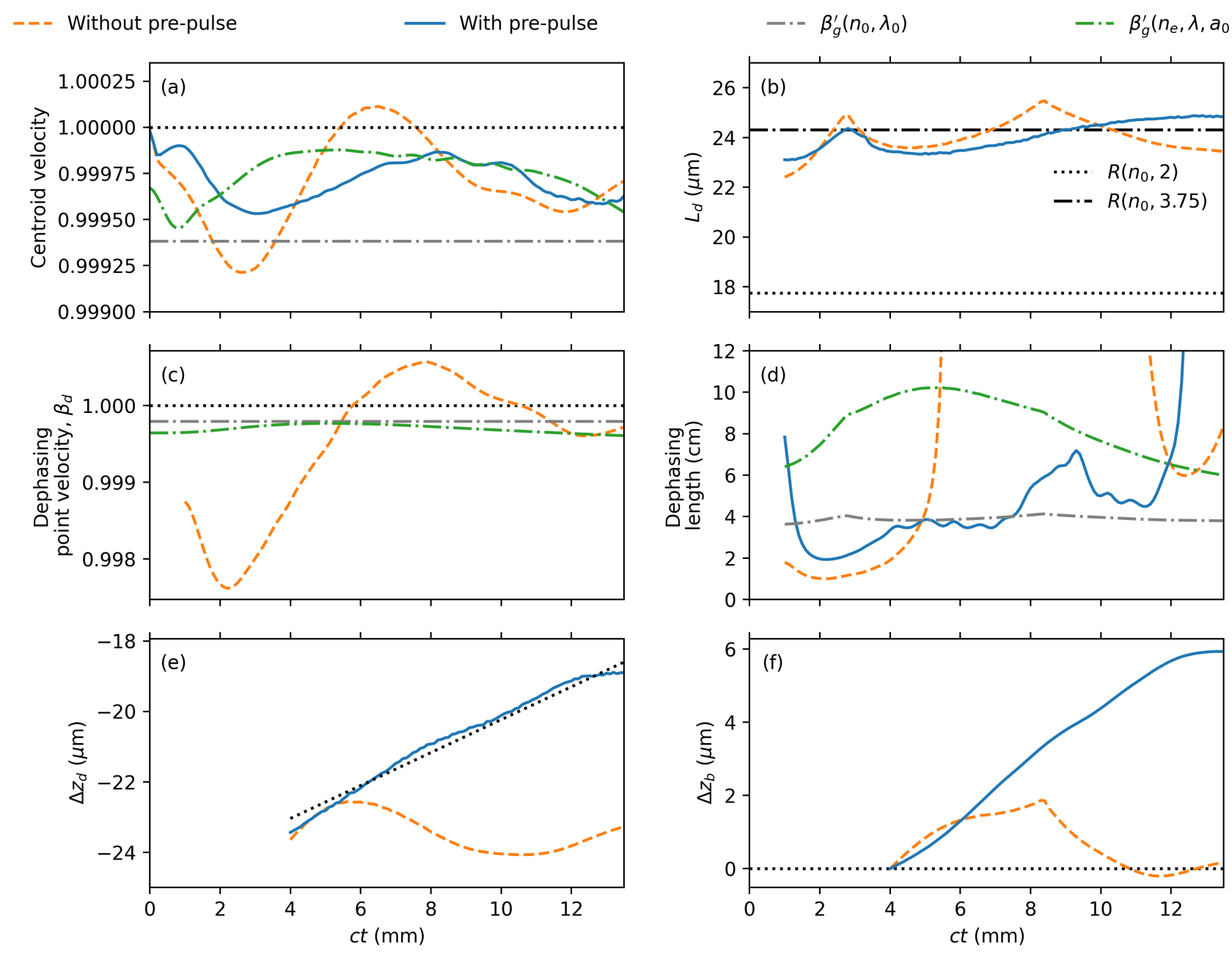

Figure 3. With (blue solid line) and without (orange dashed line) a pre-pulse. Evolution of: (a) the velocity of the laser centroid; (b) the separation between the back of the bubble and the dephasing point; (c) the velocity of the dephasing point; (d) the dephasing length; (e) the separation between electrons injected at $4 \mathrm{~mm}$ and the dephasing point; and (f) the separation electrons injected at $4 \mathrm{~mm}$ and the back of the bubble. In part (b), the estimated bubble radius $R\left(n_{0}, a_{0}\right)$ for the undisturbed plasma density $n_{0}$ [defined by equation (4)] is indicated for the initial $a_{0}=2$ by the dotted line and for the peak $a_{0}=3.75$ by the dot-dash line. In parts (a), (c) and (d) predictions based on the reduced group velocity from (2) and (8) are indicated by the grey and green dot-dash lines, respectively.

from the slope of the line $(\approx 0.47 \mu \mathrm{m} / \mathrm{mm}$, indicated by dotted line $)$ we can estimate that the electron will reach dephasing after about $5.0 \mathrm{~cm}$ giving a maximum energy of approximately $3.1 \mathrm{GeV}$. (Note that above $12 \mathrm{~mm}$ the gradient reduces, which would delay dephasing and allow for even higher energy to be reached.)

The case without the pre-pulse is completely different. The separation from the dephasing point [Fig. 3(e)] remains high, which implies that we can accelerate for longer and achieve higher energy. However, Fig. 3(f) shows the separation of the electrons from the back of the bubble, $\Delta z_{b}=z-z_{b}$. From this we observe that rapid contraction of the bubble actually causes the back of the bubble to overtake the electrons. Not only does this place the electrons in a (very high) decelerating field that reduces their energy, but it significantly reduces bunch quality. 


\section{CONCLUSIONS}

LWFAs can sustain significantly higher accelerating fields than conventional accelerators, which makes them more compact and potentially more affordable. Acceleration of self-injected electrons to very high energies has been demonstrated, but novel methods of manipulating and controlling these devices is necessary to tailor the accelerated bunches for applications.

The group velocity plays an important role in determining dephasing and the maximum obtainable electron energy is usually based on equation (1). However, significant variation of the laser intensity and plasma parameters during propagation produces a dynamically-varying accelerating structure, which expands and contracts as it responds to the variation of the laser and group velocity. This has an impact on the maximum energy achievable and on the quality of the electron bunches, which makes estimates based on the initial laser and plasmas properties unrepresentative of the system. In the LWFA, expansion and contraction of the bubble can either prolong or curtail acceleration. We have also shown how ontraction can cause the back of the bubble to catch up with and even overtake the electrons, limiting their energy and severely altering the bunch quality (such as energy spread and emittance).

The introduction of a short-duration high intensity pre-pulse just ahead of the main laser pulse prepares the plasma for the arrival of the main pulse. The pre-pulse modifies the evolution of the main laser and the wakefield that it drives, enhancing the stability of the accelerating structure and allowing higher energy and better quality bunches to be produced. The system can be optimised using the laser intensities as well as their relative separation (delay), which enables control of the LWFA accelerating structure. Moreover, as well as electron accelerators, suitable control over the wakefield bubble could allow the ion cavity to be used in ion channel lasers and plasma wigglers.

Data are openly available online from the University of Strathclyde KnowledgeBase. ${ }^{44}$

\section{ACKNOWLEDGMENTS}

SRY would like to thank Adam Noble for useful discussions. This work was supported by the U.K. EPSRC (grant number EP/N028694/1) and received funding from the European Union's Horizon 2020 research and innovation programme Laserlab Europe (grant number 871124). SRY and BE acknowledge support from the STFC (grant number ST/G008248/1). Funding was also provided by the Council of Scientific and Industrial Research and Department of Science and Technology, Govt. of India. Results were obtained using the ARCHIE-WeSt High Performance Computer (www.archie-west.ac.uk) based at the University of Strathclyde, and the facilities of the N8 Centre of Excellence in Computationally Intensive Research (N8 CIR) provided and funded by the N8 research partnership and EPSRC (grant number EP/T022167/1), co-ordinated by the Universities of Durham, Manchester and York.

\section{REFERENCES}

[1] Tajima, T. and Dawson, J. M., "Laser Electron Accelerator," Phys. Rev. Lett. 43, 267-270 (1979).

[2] Tooley, M. P., Ersfeld, B., Yoffe, S. R., Noble, A., Brunetti, E., Sheng, Z. M., Islam, M. R., and Jaroszynski, D. A., "Towards Attosecond High-Energy Electron Bunches: Controlling Self-Injection in Laser-Wakefield Accelerators Through Plasma-Density Modulation," Phys. Rev. Lett. 119, 044801 (2017).

[3] Yoffe, S. R., Ersfeld, B., Tooley, M. P., Noble, A., Fraser, R., and Jaroszynski, D. A., "Controlled generation of ultra-short electron bunches using density modulation," in [Relativistic Plasma Waves and Particle Beams as Coherent and Incoherent Radiation Sources III], Jaroszynski, D. A. and Hur, M., eds., 11036, 64-69, International Society for Optics and Photonics, SPIE (2019).

[4] Mangles, S. P. D., Murphy, C. D., Najmudin, Z., Thomas, A. G. R., Collier, J. L., Dangor, A. E., Divall, E. J., Foster, P. S., Gallacher, J. G., Hooker, C. J., Jaroszynski, D. A., Langley, A. J., Mori, W. B., Norreys, P. A., Tsung, F. S., Viskup, R., Walton, B. R., and Krushelnick, K., "Monoenergetic beams of relativistic electrons from intense laser-plasma interactions," Nature 431, 535-538 (2004).

[5] Geddes, C. G. R., Tóth, C., van Tilborg, J., Esarey, E., Schroeder, C. B., Bruhwiler, D., Nieter, C., Cary, J., and Leemans, W. P., "High-quality electron beams from a laser wakefield accelerator using plasma-channel guiding," Nature 431, 538-541 (2004). 
[6] Faure, J., Glinec, Y., Pukhov, A., Kiselev, S., Gordienko, S., Lefebvre, E., Rousseau, J.-P., Burgy, F., and Malka, V., "A laser-plasma accelerator producing monoenergetic electron beams," Nature 431, 541-544 (2004).

[7] Leemans, W. P., Nagler, B., Gonsalves, A. J., Tóth, C., Nakamura, K., Geddes, C. G. R., Esarey, E., Schroeder, C. B., and Hooker, S. M., "GeV electron beams from a centimetre-scale accelerator," Nature Physics 2, 696-699 (2006).

[8] Leemans, W., Gonsalves, A., Mao, H.-S., Nakamura, K., Benedetti, C., Schroeder, C., Tóth, C., Daniels, J., Mittelberger, D., Bulanov, S., Vay, J.-L., Geddes, C., and Esarey, E., "Multi-gev electron beams from capillary-discharge-guided subpetawatt laser pulses in the self-trapping regime," Physical Review Letters 113, 245002 (2014).

[9] Gonsalves, A., Nakamura, K., Daniels, J., Benedetti, C., Pieronek, C., de Raadt, T., Steinke, S., Bin, J., Bulanov, S., van Tilborg, J., Geddes, C., Schroeder, C., Tóth, C., Esarey, E., Swanson, K., Fan-Chiang, L., Bagdasarov, G., Bobrova, N., Gasilov, V., Korn, G., Sasorov, P., and Leemans, W., "Petawatt laser guiding and electron beam acceleration to $8 \mathrm{GeV}$ in a laser-heated capillary discharge waveguide," Physical Review Letters 122, 084801 (2019).

[10] Wiggins, S. M., Issac, R. C., Welsh, G. H., Brunetti, E., Shanks, R. P., Anania, M. P., Cipiccia, S., Manahan, G. G., Aniculaesei, C., Ersfeld, B., Islam, M. R., Burgess, R. T. L., Vieux, G., Gillespie, W. A., MacLeod, A. M., van der Geer, S. B., de Loos, M. J., and Jaroszynski, D. A., "High quality electron beams from a laser wakefield accelerator," Plasma Physics and Controlled Fusion 52(12), 124032 (2010).

[11] Brunetti, E., Shanks, R. P., Manahan, G. G., Islam, M. R., Ersfeld, B., Anania, M. P., Cipiccia, S., Issac, R. C., Raj, G., Vieux, G., Welsh, G. H., Wiggins, S. M., and Jaroszynski, D. A., "Low emittance, high brilliance relativistic electron beams from a laser-plasma accelerator," Physical Review Letters 105, 215007 (2010).

[12] Lundh, O., Lim, J., Rechatin, C., Ammoura, L., Ben-Ismaïl, A., Davoine, X., Gallot, G., Goddet, J.-P., Lefebvre, E., Malka, V., and Faure, J., "Few femtosecond, few kiloampere electron bunch produced by a laser-plasma accelerator," Nature Physics 7, 219-222 (2011).

[13] Islam, M. R., Brunetti, E., Shanks, R. P., Ersfeld, B., Issac, R. C., Cipiccia, S., Anania, M.-P., Welsh, G. H., Wiggins, S. M., Noble, A., Cairns, R. A., Raj, G., and Jaroszynski, D. A., "Near-threshold electron injection in the laser-plasma wakefield accelerator leading to femtosecond bunches," New Journal of Physics 17, 093033 (2015).

[14] Rousse, A., Phuoc, K. T., Shah, R., Pukhov, A., Lefebvre, E., Malka, V., Kiselev, S., Burgy, F., Rousseau, J.-P., Umstadter, D., and Hulin, D., "Production of a keV X-Ray Beam from Synchrotron Radiation in Relativistic Laser-Plasma Interaction," Phys. Rev. Lett. 93, 135005 (2004).

[15] Cipiccia, S., Islam, M. R., Ersfeld, B., Shanks, R. P., Brunetti, E., Vieux, G., Yang, X., Issac, R. C., Wiggins, S. M., Welsh, G. H., Anania, M.-P., Maneuski, D., Montgomery, R., Smith, G., Hoek, M., Hamilton, D. J., Lemos, N. R. C., Symes, D., Rajeev, P. P., Shea, V. O., Dias, J. M., and Jaroszynski, D. A., "Gamma-rays from harmonically resonant betatron oscillations in a plasma wake," Nature Physics 7, 867-871 (2011).

[16] Wenz, J., Schleede, S., Khrennikov, K., Bech, M., Thibault, P., Heigoldt, M., Pfeiffer, F., and Karsch, S., "Quantitative x-ray phase-contrast microtomography from a compact laser-driven betatron source," Nature Communications 6, 7568 (2015).

[17] Cole, J. M., Wood, J. C., Lopes, N. C., Poder, K., Abel, R. L., Alatabi, S., Bryant, J. S. J., Jin, A., Kneip, S., Mecseki, K., Symes, D. R., Mangles, S. P. D., and Najmudin, Z., "Laser-wakefield accelerators as hard x-ray sources for 3d medical imaging of human bone," Scientific Reports 5, 13244 (2015).

[18] Cole, J. M., Symes, D. R., Lopes, N. C., Wood, J. C., Poder, K., Alatabi, S., Botchway, S. W., Foster, P. S., Gratton, S., Johnson, S., Kamperidis, C., Kononenko, O., De Lazzari, M., Palmer, C. A. J., Rusby, D., Sanderson, J., Sandholzer, M., Sarri, G., Szoke-Kovacs, Z., Teboul, L., Thompson, J. M., Warwick, J. R., Westerberg, H., Hill, M. A., Norris, D. P., Mangles, S. P. D., and Najmudin, Z., "High-resolution $\mu$ ct of a mouse embryo using a compact laser-driven x-ray betatron source," Proceedings of the National Academy of Sciences 115, 6335-6340 (2018).

[19] Fuchs, T., Szymanowski, H., Oelfke, U., Glinec, Y., Rechatin, C., Faure, J., and Malka, V., "Treatment planning for laser-accelerated very-high energy electrons," Physics in Medicine and Biology 54, 3315-3328 (2009). 
[20] Subiel, A., Moskvin, V., Welsh, G. H., Cipiccia, S., Reboredo, D., DesRosiers, C., and Jaroszynski, D. A., "Challenges of dosimetry of ultra-short pulsed very high energy electron beams," Physica Medica 42, 327-331 (2017).

[21] Yang, X., Brunetti, E., and Jaroszynski, D. A., "High-energy coherent terahertz radiation emitted by wideangle electron beams from a laser-wakefield accelerator," New Journal of Physics 20, 043046 (2018).

[22] Kokurewicz, K., Brunetti, E., Welsh, G. H., Wiggins, S. M., Boyd, M., Sorensen, A., Chalmers, A. J., Schettino, G., Subiel, A., DesRosiers, C., and Jaroszynski, D. A., "Focused very high-energy electron beams as a novel radiotherapy modality for producing high-dose volumetric elements," Scientific Reports $\mathbf{9}, 10837$ (2019).

[23] Jaroszynski, D. A. and Vieux, G., "Coherent Radiation Sources Based on Laser Plasma Accelerators," AIP Conference Proceedings 647, 902-914 (2002).

[24] Schlenvoigt, H.-P., Haupt, K., Debus, A., Budde, F., Jäckel, O., Pfotenhauer, S., Schwoerer, H., Rohwer, E., Gallacher, J. G., Brunetti, E., Shanks, R. P., Wiggins, S. M., and Jaroszynski, D. A., "A compact synchrotron radiation source driven by a laser-plasma wakefield accelerator," Nature Physics 4, 130-133 (2008).

[25] Anania, M.-P., Brunetti, E., Wiggins, S. M., Grant, D. W., Welsh, G. H., Issac, R. C., Cipiccia, S., Shanks, R. P., Manahan, G. G., Aniculaesei, C., van der Geer, S. B., de Loos, M. J., Poole, M. W., Shepherd, B. J. A., Clarke, J. A., Gillespie, W. A., MacLeod, A. M., and Jaroszynski, D. A., "An ultrashort pulse ultra-violet radiation undulator source driven by a laser plasma wakefield accelerator," Applied Physics Letters 104, 264102 (2014).

[26] Whittum, D. H., Sessler, A. M., and Dawson, J. M., "Ion-channel laser," Phys. Rev. Lett. 64, 2511-2514 (1990).

[27] Ersfeld, B., Bonifacio, R., Chen, S., Islam, M. R., Smorenburg, P. W., and Jaroszynski, D. A., "The ion channel free-electron laser with varying betatron amplitude," New Journal of Physics 16, 093025 (2014).

[28] Lehe, R., Kirchen, M., Andriyash, I. A., Godfrey, B. B., and Vay, J. L., "A spectral, quasi-cylindrical and dispersion-free Particle-In-Cell algorithm," Computer Physics Communications 203, 66-82 (2016).

[29] Mendonca, J. T., [Theory of Photon Acceleration], Institute of Physics Publishing, Bristol, UK (2001).

[30] Tooley, M. P., Self Injection and Radiation Production in Laser Wakefield Accelerators, PhD thesis, University of Strathclyde, UK (2020).

[31] Benedetti, C., Schroeder, C. B., Esarey, E., and Leemans, W. P., "Quasi-matched propagation of ultra-short, intense laser pulses in plasma channels," Physics of Plasmas 19, 053101 (2012).

[32] Khachatryan, A. G., Irman, A., van Goor, F. A., and Boller, K.-J., "Femtosecond electron-bunch dynamics in laser wakefields and vacuum," Phys. Rev. ST Accel. Beams 10, 121301 (2007).

[33] Durfee, C. G. and Milchberg, H. M., "Light pipe for high intensity laser pulses," Phys. Rev. Lett. 71, 2409-2412 (1993).

[34] Clark, T. R. and Milchberg, H. M., "Time- and space-resolved density evolution of the plasma waveguide," Phys. Rev. Lett. 78, 2373-2376 (1997).

[35] Nikitin, S. P., Alexeev, I., Fan, J., and Milchberg, H. M., "High efficiency coupling and guiding of intense femtosecond laser pulses in preformed plasma channels in an elongated gas jet," Phys. Rev. E 59, R3839R3842 (1999).

[36] Volfbeyn, P., Esarey, E., and Leemans, W. P., "Guiding of laser pulses in plasma channels created by the ignitor-heater technique," Physics of Plasmas 6, 2269-2277 (1999).

[37] Xiao, Y.-F., Chu, H.-H., Tsai, H.-E., Lee, C.-H., Lin, J.-Y., Wang, J., and Chen, S.-Y., "Efficient generation of extended plasma waveguides with the axicon ignitor-heater scheme," Physics of Plasmas 11, L21-L24 (2004).

[38] Kumarappan, V., Kim, K. Y., and Milchberg, H. M., "Guiding of intense laser pulses in plasma waveguides produced from efficient, femtosecond end-pumped heating of clustered gases," Phys. Rev. Lett. 94, 205004 (2005).

[39] Lemos, N., Grismayer, T., Cardoso, L., Geada, J., Figueira, G., and Dias, J. M., "Effects of laser polarization in the expansion of plasma waveguides," Physics of Plasmas 20(10), 103109 (2013). 
[40] Lemos, N., Grismayer, T., Cardoso, L., Figueira, G., Issac, R., Jaroszynski, D. A., and Dias, J. M., "Plasma expansion into a waveguide created by a linearly polarized femtosecond laser pulse," Physics of Plasmas 20(6), 063102 (2013).

[41] Ge, Z. Y., Yin, Y., Li, S. X., Yu, M. Y., Yu, T. P., Xu, H., Zhuo, H. B., Ma, Y. Y., Shao, F. Q., and Tian, C. L., "Enhancement of electron injection in laser wakefield acceleration using auxiliary interfering pulses," New Journal of Physics 14, 103015 (2012).

[42] Hooker, S. M., "Developments in laser-driven plasma accelerators," Nature Photonics 7, 775-782 (2013).

[43] Hooker, S. M., Bartolini, R., Mangles, S. P. D., Tünnermann, A., Corner, L., Limpert, J., Seryi, A., and Walczak, R., "Multi-pulse laser wakefield acceleration: a new route to efficient, high-repetition-rate plasma accelerators and high flux radiation sources," Journal of Physics B: Atomic, Molecular and Optical Physics 47, 234003 (2014).

[44] "Data for: 'Group velocity control in the LWFA using a pre-pulse'." DOI: 10.15129/4f2af876-c1e0-4e11aec6-87c55fa84f76 (2021). 